\title{
Visible Points on Curves over Finite Fields
}

\author{
by \\ Igor E. SHPARLINSKI and José Felipe VOLOCH
}

Presented by Andrzej SCHINZEL

Summary. For a prime $p$ and an absolutely irreducible modulo $p$ polynomial $f(U, V) \in$ $\mathbb{Z}[U, V]$ we obtain an asymptotic formula for the number of solutions to the congruence $f(x, y) \equiv a(\bmod p)$ in positive integers $x \leq X, y \leq Y$, with the additional condition $\operatorname{gcd}(x, y)=1$. Such solutions have a natural interpretation as solutions which are visible from the origin. These formulas are derived on average over $a$ for a fixed prime $p$, and also on average over $p$ for a fixed integer $a$.

1. Introduction. Let $p$ be a prime and let $f(U, V) \in \mathbb{Z}[U, V]$ be a bivariate polynomial with integer coefficients.

For real $X$ and $Y$ with $1 \leq X, Y \leq p$ and an integer $a$ we consider the set

$$
\mathcal{F}_{p, a}(X, Y)=\{(x, y) \in[1, X] \times[1, Y]: f(x, y) \equiv a(\bmod p)\}
$$

which is the set of points on level curves of $f(U, V)$ modulo $p$.

If $f(x, y)-a$ is a nonconstant absolutely irreducible polynomial modulo $p$ of degree at least 2, then one can easily derive from the Bombieri bound [2] that

$$
\# \mathcal{F}_{p, a}(X, Y)=\frac{X Y}{p}+O\left(p^{1 / 2}(\log p)^{2}\right),
$$

where the implied constant depends only on $\operatorname{deg} f$ (see, e.g., $[3,4,9,11]$ ).

In this paper we consider an apparently new question of studying the cardinality of the set

$$
N_{p, a}(X, Y)=\#\left\{(x, y) \in \mathcal{F}_{p, a}(X, Y): \operatorname{gcd}(x, y)=1\right\} .
$$

These points have a natural geometric interpretation as points on $\mathcal{F}_{p, a}(X, Y)$

2000 Mathematics Subject Classification: 11A07, 11K38, 11L40. mial.

Key words and phrases: points visible from the origin, absolutely irreducible polyno- 
which are "visible" from the origin (see $[1,6,7,10]$ and references therein for several other aspects of distribution of visible points in various regions).

We show that on average over $a=0, \ldots, p-1$, the cardinality $N_{p, a}(X, Y)$ is close to its expected value $6 X Y / \pi^{2} p$ whenever

$$
X Y \geq p^{3 / 2+\varepsilon}
$$

for any fixed $\varepsilon>0$ and sufficiently large $p$.

We then consider the dual situation, when $a$ is fixed (in particular we take $a=0$ ) but $p$ varies through all primes up to $T$.

Our approach is based on a rather straightforward application of the inclusion-exclusion formula involving the Möbius function. We apply (1) to the lower terms of this formula which leads to the main term. However, the main difficulty is in getting a nontrivial estimate for the tail terms. This is exactly where we need to introduce some averaging in order to get such a nontrivial bound.

We recall $A \ll B$ and $A=O(B)$ both mean that $|A| \leq c B$ holds with some constant $c>0$, which may depend on some specified set of parameters.

2. Absolute irreducibility of level curves. We start with the following statement which could be of independent interest.

Lemma 1. If $F(U, V) \in \mathbb{K}[U, V]$ is absolutely irreducible of degree $n$ over a field $\mathbb{K}$, then $F(U, V)-a$ is absolutely irreducible for all but at most $C(n)$ elements $a \in \mathbb{K}$, where $C(n)$ depends only on $n$.

Proof. The set of polynomials of degree $n$ is parametrized by a projective space $\mathbb{P}^{s(n)}$ of dimension $s(n)=(n+1)(n+2) / 2$ over $\mathbb{K}$, coordinatized by the coefficients. The subset $X$ of $\mathbb{P}^{k(n)}$ consisting of reducible polynomials is a Zariski closed subset because it is the union of the images of the maps

$$
\mathbb{P}^{s(k)} \times \mathbb{P}^{s(n-k)} \rightarrow \mathbb{P}^{s(n)}, \quad k \leq n / 2,
$$

given by multiplying a polynomial of degree $k$ with a polynomial of degree $n-k$. The map $t \mapsto F(U, V)-t$ describes a line in $\mathbb{P}^{s(n)}$ and by the assumption of absolute irreducibility of $F$, this line is not contained in $X$. So, by the Bézout theorem, it meets $X$ in at most $C(n)$ points, where $C(n)$ is the degree of $X$. Hence for all but at most $C(n)$ values of $a, F(U, V)-a$ is absolutely irreducible.

3. Visible points on almost all level curves. Throughout this section, the implied constants in the notations $A \ll B$ and $A=O(B)$ may depend on the degree $n=\operatorname{deg} f$.

THEOREM 2. Let $f$ be a polynomial with integer coefficients which is absolutely irreducible and of degree greater than one modulo the prime $p$. 
Then for real $X$ and $Y$ with $1 \leq X, Y \leq p$ we have

$$
\sum_{a=0}^{p-1}\left|N_{p, a}(X, Y)-\frac{6}{\pi^{2}} \cdot \frac{X Y}{p}\right| \ll X^{1 / 2} Y^{1 / 2} p^{3 / 4} \log p .
$$

Proof. Let $\mathcal{A}_{p}$ consist of $a \in\{0, \ldots, p-1\}$ for which $f(U, V)-a$ is absolutely irreducible modulo $p$.

For an integer $d$, we define

$$
M_{p, a}(d ; X, Y)=\#\left\{(x, y) \in \mathcal{F}_{p, a}(X, Y) \mid \operatorname{gcd}(x, y) \equiv 0(\bmod d)\right\} .
$$

Let $\mu(d)$ denote the Möbius function. We recall that $\mu(1)=1, \mu(d)=0$ if $d \geq 2$ is not square-free and $\mu(d)=(-1)^{\omega(d)}$ otherwise, where $\omega(d)$ is the number of distinct prime divisors of $d$. By the inclusion-exclusion principle, we write

$$
N_{p, a}(X, Y)=\sum_{d=1}^{\infty} \mu(d) M_{p, a}(d ; X, Y)
$$

Writing

$$
x=d s \quad \text { and } \quad y=d t,
$$

we have

$$
M_{p, a}(d ; X, Y)=\#\{(s, t) \in[1, X / d] \times[1, Y / d] \mid f(d s, d t) \equiv a(\bmod p)\} .
$$

Thus $M_{p, a}(d ; X, Y)$ is the number of points on a curve in a given box. If $a \in \mathcal{A}_{p}$ and $1 \leq d<p$ then $f(d U, d V)-a$ remains absolutely irreducible modulo $p$. Accordingly, we have an analogue of (1) which asserts that

$$
M_{p, a}(d ; X, Y)=\frac{X Y}{d^{2} p}+O\left(p^{1 / 2}(\log p)^{2}\right)
$$

We fix some positive parameter $D<p$ and substitute the bound (4) in (3) for $d \leq D$, getting

$$
\begin{aligned}
& N_{p, a}(X, Y) \\
& =\sum_{d \leq D}\left(\frac{\mu(d) X Y}{d^{2} p}+O\left(p^{1 / 2}(\log p)^{2}\right)\right)+O\left(\sum_{d>D} M_{p, a}(d ; X, Y)\right) \\
& =\frac{X Y}{p} \sum_{d \leq D} \frac{\mu(d)}{d^{2}}+O\left(D p^{1 / 2}(\log p)^{2}+\sum_{d>D} M_{p, a}(d ; X, Y)\right)
\end{aligned}
$$

for every $a \in \mathcal{A}_{p}$.

Furthermore

$$
\sum_{d \leq D} \frac{\mu(d)}{d^{2}}=\sum_{d=1}^{\infty} \frac{\mu(d)}{d^{2}}+O\left(D^{-1}\right)=\prod_{l}\left(1-\frac{1}{l^{2}}\right)+O\left(D^{-1}\right)
$$


where the product is taken over all prime numbers $l$. Recalling that

$$
\prod_{l}\left(1-\frac{1}{l^{2}}\right)=\zeta(2)^{-1}=\frac{6}{\pi^{2}}
$$

(see [5, Equation (17.2.2) and Theorem 280]), we obtain

$$
\left|N_{p, a}(X, Y)-\frac{6}{\pi^{2}} \cdot \frac{X Y}{p}\right| \ll \frac{X Y}{D p}+D p^{1 / 2}(\log p)^{2}+\sum_{d>D} M_{p, a}(d ; X, Y)
$$

for every $a \in \mathcal{A}_{p}$.

We also remark that

$$
\begin{aligned}
\sum_{a=0}^{p-1} \sum_{d>D} M_{p, a}(d ; X, Y) & =\sum_{d>D} \sum_{a=0}^{p-1} M_{p, a}(d ; X, Y) \\
& =\sum_{d>D}\left\lfloor\frac{X}{d}\right\rfloor\left\lfloor\frac{Y}{d}\right\rfloor \leq X Y \sum_{d>D} \frac{1}{d^{2}} \ll X Y / D .
\end{aligned}
$$

Therefore, using the bounds (5) and (6), we obtain

$$
\sum_{a \in \mathcal{A}_{p}}\left|N_{p, a}(X, Y)-\frac{6}{\pi^{2}} \cdot \frac{X Y}{p}\right| \ll X Y / D+D p^{3 / 2}(\log p)^{2} .
$$

For $a \notin \mathcal{A}_{p}$ we estimate $N_{p, a}(X, Y)$ trivially as

$$
N_{p, a}(X, Y) \leq \min \{X, Y\} \operatorname{deg} f \ll \sqrt{X Y} .
$$

Thus by Lemma 1,

$$
\sum_{a \notin \mathcal{A}_{p}}\left|N_{p, a}(X, Y)-\frac{6}{\pi^{2}} \cdot \frac{X Y}{p}\right| \ll \max \{\sqrt{X Y}, X Y / p\} \ll \sqrt{X Y} .
$$

Combining (7) and (8) and taking $D=X^{1 / 2} Y^{1 / 2} p^{-3 / 4}(\log p)^{-1}$ we conclude the proof.

COROLlary 3. Let $f$ be a polynomial with integer coefficients which is absolutely irreducible and of degree greater than one modulo the prime $p$. If $X Y \geq p^{3 / 2}(\log p)^{2+\varepsilon}$ for some fixed $\varepsilon>0$, then

$$
N_{p, a}(X, Y)=\left(\frac{6}{\pi^{2}}+o(1)\right) \frac{X Y}{p}
$$

for all but $o(p)$ values of $a=0, \ldots, p-1$.

4. Visible points on almost all reductions. Throughout this section, the implied constants in the notations $A \ll B$ and $A=O(B)$ may depend on the coefficients of $f$.

To simplify notation we put

$$
\mathcal{F}_{p}(X, Y)=\mathcal{F}_{p, 0}(X, Y) \quad \text { and } \quad N_{p}(X, Y)=N_{p, 0}(X, Y) .
$$


We only consider polynomials $f$ with integer coefficients such that the equation $f(x, y)=0$ has only finitely many integer solutions. We recall that the Siegel theorem guarantees this for a very general class of polynomials.

THEOREM 4. Let $f$ be a polynomial with integer coefficients which is absolutely irreducible and of degree greater than one such that the equation $f(x, y)=0$ has only finitely many integer solutions. Then for real $T, X$ and $Y$ such that $T \geq 2 \max (X, Y)$ and $X Y \geq T^{3 / 2} \log T$ we have

$$
\sum_{T / 2 \leq p \leq T}\left|N_{p}(X, Y)-\frac{6}{\pi^{2}} \cdot \frac{X Y}{p}\right| \ll X^{1 / 2} Y^{1 / 2} T^{3 / 4}(\log T)^{3 / 2}
$$

as $T \rightarrow \infty$, where the sum is taken over all primes $p$ with $T / 2 \leq p \leq T$.

Proof. It is enough to consider $T$ large enough so that $f$ remains absolutely irreducible and of degree greater than one for all $p, T / 2 \leq p \leq T$. As before we have

$$
\left|N_{p}(X, Y)-\frac{6}{\pi^{2}} \cdot \frac{X Y}{p}\right| \ll \frac{X Y}{D p}+D p^{1 / 2}(\log p)^{2}+\sum_{d>D} M_{p}(d ; X, Y)
$$

where

$$
M_{p}(d ; X, Y)=\#\left\{(x, y) \in \mathcal{F}_{p}(X, Y) \mid \operatorname{gcd}(x, y) \equiv 0(\bmod d)\right\} .
$$

We also remark that

$$
\begin{aligned}
\sum_{T / 2 \leq p \leq T} \sum_{d>D} M_{p}(d ; X, Y) & =\sum_{d>D} \sum_{T / 2 \leq p \leq T} M_{p}(d ; X, Y) \\
& =\sum_{d>D} \sum_{1 \leq s \leq X / d} \sum_{\substack { 1 \leq t \leq Y / d \\
\begin{subarray}{c}{T / 2 \leq p \leq T \\
p \mid f(d s, d t){ 1 \leq t \leq Y / d \\
\begin{subarray} { c } { T / 2 \leq p \leq T \\
p | f ( d s , d t ) } }\end{subarray}} 1 .
\end{aligned}
$$

Let $\mathcal{Z}$ be the set of integer zeros $(x, y)$ of $f(x, y)=0$. We assume that $D$ is large enough so that

$$
f(d s, d t) \neq 0
$$

for $d>D$ and $s, t \geq 1$.

As before, we denote by $\omega(k)$ the number of prime divisors of a positive integer $k$ and note that $\omega(k) \ll \log k$. Thus for $(u, v) \notin \mathcal{Z}$ we can estimate the inner sum over $p$ in $(10)$ as $\omega(|f(d s, d t)|) \ll \log (X Y) \ll \log T$. Therefore

$$
\begin{aligned}
\sum_{T / 2 \leq p \leq T} \sum_{d>D} M_{p}(d ; X, Y) & \leq \sum_{d>D} \sum_{\substack{1 \leq s \leq X / d \\
1 \leq t \leq Y / d}} \sum_{p \mid f(d s, d t)} 1 \\
& \leq \sum_{d>D} \sum_{\substack{1 \leq s \leq X / d \\
1 \leq t \leq Y / d}} \log T \ll X Y D^{-1} \log T .
\end{aligned}
$$

We also note that by the prime number theorem, 


$$
\sum_{T / 2 \leq p \leq T} \frac{1}{p} \leq \frac{2}{T} \sum_{T / 2 \leq p \leq T} 1 \ll \frac{1}{\log T} .
$$

We now put everything together getting

$$
\begin{aligned}
\sum_{T / 2 \leq p \leq T}\left|N_{p}(X, Y)-\frac{6}{\pi^{2}} \cdot \frac{X Y}{p}\right| & \ll \frac{X Y}{D \log T}+D T^{3 / 2}(\log T)^{2}+\frac{X Y \log T}{D} \\
& \ll D T^{3 / 2}(\log T)^{2}+\frac{X Y \log T}{D} .
\end{aligned}
$$

We now take $D=c X^{1 / 2} Y^{1 / 2} T^{-3 / 4}(\log T)^{-1 / 2}$ for a sufficiently large constant $c$ depending only on $f$ (to guarantee that we have (11) for $d>D$ and $s, t \geq 1$ ), which yields the result.

COROLlary 5. Let $f$ be a polynomial with integer coefficients which is absolutely irreducible and of degree greater than one such that the equation $f(x, y)=0$ has only finitely many integer solutions. If $T \geq 2 \max (X, Y)$ and $X Y \geq T^{3 / 2+\varepsilon}$ for some fixed $\varepsilon>0$, then

$$
N_{p}(X, Y)=\left(\frac{6}{\pi^{2}}+o(1)\right) \frac{X Y}{p}
$$

for all but $o(T / \log T)$ primes $p \in[T / 2, T]$.

5. Remarks. Certainly it is interesting to obtain an asymptotic formula for $N_{p, a}(X, Y)$ which holds for every $a$. Even the case of $X=Y=p$ is of interest. We remark that for the polynomial $f(U, V)=U V$ such an asymptotic formula is given in [8] and is nontrivial provided $X Y \geq p^{3 / 2+\varepsilon}$ for some fixed $\varepsilon>0$. However, the technique of [8] does not seem to apply to more general polynomials.

We remark that studying such special cases as visible points on the curves of the shape $f(U, V)=V-g(U)$ (corresponding to points on the graph of a univariate polynomial) and $f(U, V)=V^{2}-X^{3}-r X-s$ (corresponding to points on an elliptic curve) is also of interest and may be more accessible than the general case.

Acknowledgements. This work began during a pleasant visit by I. S. to University of Texas sponsored by NSF grant DMS-05-03804; the support and hospitality of this institution are gratefully acknowledged. During the preparation of this paper, I. S. was supported in part by ARC grant DP0556431.

\section{References}

[1] F. P. Boca, C. Cobeli and A. Zaharescu, Distribution of lattice points visible from the origin, Comm. Math. Phys. 213 (2000), 433-470. 
[2] E. Bombieri, On exponential sums in finite fields, Amer. J. Math. 88 (1966), 71-105.

[3] C. Cobeli and A. Zaharescu, On the distribution of the $\mathbb{F}_{p}$-points on an affine curve in $r$ dimensions, Acta Arith. 99 (2001), 321-329.

[4] A. Granville, I. E. Shparlinski and A. Zaharescu, On the distribution of rational functions along a curve over $\mathbb{F}_{p}$ and residue races, J. Number Theory 112 (2005), $216-237$.

[5] G. H. Hardy and E. M. Wright, An Introduction to the Theory of Numbers, The Clarendon Press, Oxford Univ. Press, New York, 1979.

[6] M. N. Huxley and W. G. Nowak, Primitive lattice points in convex planar domains, Acta Arith. 76 (1996), 271-283.

[7] W. G. Nowak, Primitive lattice points inside an ellipse, Czechoslovak Math. J. 55 (2005), 519-530.

[8] I. E. Shparlinski, Primitive points on a modular hyperbola, Bull. Polish Acad. Sci. Math. 54 (2006), 193-200.

[9] M. Vajaitu and A. Zaharescu, Distribution of values of rational maps on the $\mathbb{F}_{p^{-}}$ points on an affine curve, Monatsh. Math. 136 (2002), 81-86.

[10] W. G. Zhai, On primitive lattice points in planar domains, Acta Arith. 109 (2003), $1-26$.

[11] Z. Y. Zheng, The distribution of zeros of an irreducible curve over a finite field, J. Number Theory 59 (1996), 106-118.

Igor E. Shparlinski

Department of Computing

Macquarie University

Sydney, NSW 2109, Australia

E-mail: igor@ics.mq.edu.au
José Felipe Voloch

Department of Mathematics

University of Texas

Austin, TX 78712, U.S.A.

E-mail: voloch@math.utexas.edu

Received May 14, 2007;

received in final form June 7, 2007 